\title{
Concomitant mycotic abdominal aortic aneurysm and lumbar tuberculous spondylitis with cauda equina syndrome: a rare condition - a case report and literature review
}

\author{
Tinnakorn Pluemvitayaporn ${ }^{1} \cdot$ Sarun Jindahra ${ }^{1,2} \cdot$ Warongporn Pongpinyopap $^{1,3} \cdot$ Sombat Kunakornsawat $^{1}$. \\ Chaiyot Thiranon $^{2} \cdot$ Weerasak Singhatanadgige $^{4} \cdot$ Apinan Uthaipaisanwong $^{5}$
}

Received: 25 October 2017 / Revised: 30 December 2017 / Accepted: 7 January 2018

(c) International Spinal Cord Society 2018

\begin{abstract}
Introduction Concomitant mycotic abdominal aortic aneurysm and lumbar tuberculous spondylitis with psoas abscess and cauda equina syndrome is extremely rare. This condition can cause serious life-threatening problems if not diagnosed and treated properly.

Case presentation We report an unusual case of a 79-year-old Thai male, who was diagnosed with concomitant mycotic abdominal aortic aneurysm and lumbar tuberculous spondylitis at the L2-L3 level with left psoas abscess and cauda equina syndrome. The surgical plan was radical surgical debridement via transpsoas approach and the defect was filled with iliac crest strut graft and posterior decompressive laminectomy and fusion with a pedicle screws and rods system. During the operation, an abdominal aortic aneurysm was iatrogenically ruptured and then was emergently treated with endovascular stent graft implantation. Subsequently, hemostasis was achieved and the patient remained hemodynamically stable. A few days later, he underwent posterior decompressive laminectomy L2-L3, fusion and instrumentation with a pedicle screws and rods system at T11-L5. After surgery, the patient recovered well and his motor power improved gradually. He was continually treated with anti-tuberculous chemotherapy for 12 months.

Discussion Concomitant mycotic aortic aneurysm and lumbar tuberculous spondylitis with psoas abscess and cauda equina syndrome is an extremely rare condition that requires prompt diagnosis and management. Its consequences can lead to serious complications such as permanent neurological damage, paralysis or even death, if left untreated. The aims of the treatment are to eradicate infection, to prevent further neurological compromise, to stabilize the spine and to protect the aortic aneurysm from rupture.
\end{abstract}

Tinnakorn Pluemvitayaporn

dr.tinnakorn@gmail.com

1 Spine Unit, Institute of Orthopaedics, Lerdsin Hospital, Bangkok, Thailand

2 Department of Orthopaedic Surgery, Sirindhorn Hospital, Bangkok, Thailand

3 Department of Orthopaedic Surgery, Pakchong Nana Hospital, Nakhon Ratchasima, Thailand

4 Department of Orthopaedic Surgery, Faculty of Medicine, Chulalongkorn University and King Chulalongkorn Memorial Hospital, Thai Red Cross Society, Bangkok, Thailand

5 Department of Surgery, Faculty of Medicine, Chulalongkorn University and King Chulalongkorn Memorial Hospital, Bangkok, Thailand

\section{Introduction}

In general, spinal infections are relatively rare, accounting for only $2-4 \%$ of all osteomyelitis infections, and mortality is estimated to be $1-20 \%$, depending on the patient group and the infecting agent [1]. In addition, tuberculous spondylitis, also known as Pott's disease, was first described in 1779 by Dr. Percival Pott [2]. It accounts for 50\% of all cases of skeletal tuberculosis. The most common site is thoracolumbar junction, followed by the lumbar region. The incidence of neurologic complications in tuberculous spondylitis varies from 10 to $43 \%$ [2, 3]. Delay in the diagnosis and treatment can lead to serious consequences such as permanent neurological deterioration, paralysis, bowel, and bladder dysfunction or cauda equina syndrome; moreover, it can be fatal if left untreated. 
Mycotic or infected aortic aneurysm, which is defined as an infectious break in the wall of an artery with formation of a blind, saccular outpouching that is contiguous with the arterial lumen $[4,5]$, is also uncommon. The incidence of infected aortic aneurysm in patients with thoracoabdominal or abdominal aneurysm is $0.7-1 \%$ [6]. This rare disease is also presents treatment difficulties and is associated with significant mortality. The mortality rate ranges from 16 to $44 \%$ [7]. The combination of these conditions are extremely rare. It can cause life-threatening problems if not diagnosed and treated properly. Therefore, the objectives of this study are to report this rare condition and to review the literature.

\section{Case presentation}

A 79-year-old Thai male was transferred and admitted to our institution with the chief complaint of severe backache for 5 months. The patient also had symptoms of low-grade fever, malaise, and significant weight loss. The symptoms were aggravated at night and could not be relieved by rest or oral analgesia. During this period, his symptoms did not significantly resolve; moreover, he had rapidly progressive weakness of both lower extremities and difficulty walking. The patient developed clinical signs of areflexic bowel and bladder function (unawareness of the need to empty the bladder, inability to prevent urinary and stool leakage, and loose anal sphincter tone) and was subsequently referred to our institution. He had no history of tuberculosis. Physical examination revealed generalized bilateral lower extremity weakness, most marked in the left side (Table 1). Sensory examination showed hypoesthesia in the distribution of the bilateral L2 dermatomes (AIS category C; motor incomplete

Table 1 revealed comparison of motor power grading between preoperative surgery and postoperative surgery

\begin{tabular}{lll}
\hline Motor & Right & Left \\
\hline Postoperative: Motor power & 2 & \\
L2: Hip flexion & 2 & 0 \\
L3: Knee extension & 2 & 0 \\
L4: Ankle dorsiflexion & 2 & 0 \\
L5: Big toe dorsiflexion & 2 & 0 \\
S1: Ankle plantarflexion & Loose tone & 0 \\
S2: Anal sphincter & & \\
Postoperative: Motor power & 4 & 3 \\
L2: Hip flexion & 3 & 3 \\
L3: Knee extension & 2 & 2 \\
L4: Ankle dorsiflexion & 2 & 2 \\
L5: Big toe dorsiflexion & 2 & 1 \\
S1: Ankle plantarflexion & Decreased tone & \\
S2: Anal sphincter & & \\
\hline
\end{tabular}

with a level of injury of L1) [8, 9]. Normoreflexia was observed. Laboratory findings revealed elevations of white blood cell count and C-reactive protein (CRP). Blood culture result was negative and tumor markers including CA19-9, prostate-specific antigen (PSA), carcinoembryonic antigen (CEA), and alpha-fetoprotein (AFP) were unremarkable. Blood for QuantiFERON-TB Gold assay test was positive. Plain radiographs of lumbosacral spine revealed an osteolytic lesion with superior endplate destruction of L3 vertebra and vertebral collapse of L2 and L3 vertebral bodies. No abnormal paravertebral soft tissue shadow was demonstrated. A calcified abdominal aorta was seen (Fig. 1), however, chest $\mathrm{x}$-ray was unremarkable. Computerized tomographic scan demonstrated a $37 \mathrm{~mm}$ false aneurysm of the infrarenal aorta surrounded by large abscess involving the left psoas muscle with osteolytic lesions at L2 and L3 levels. Computerized tomographic angiography confirmed a false aneurysm arising from the lateral wall of the aorta, $30 \mathrm{~mm}$ below origin of the renal artery and 48- $\mathrm{mm}$ above aortic bifurcation. Magnetic resonance imaging of lumbosacral spine demonstrated abnormal marrow signal intensity of L2 and L3 vertebral bodies with vertebral bodyintervertebral disc abscess formation and subligamentous spreading from L1 to L4 vertebra. A large paravertebral abscess with posterior and posterolateral extension infiltrated into the left psoas muscle from L1 to L4. This pathology had a pressure effect onto the thecal sac, compression to the both exiting nerve roots of L2 and both traversing nerve roots of L3 (Fig. 2). The diagnosis was concomitant mycotic infrarenal abdominal aortic aneurysm and lumbar tuberculous spondylitis at L2-L3 levels with left psoas abscess formation and cauda equina syndrome.

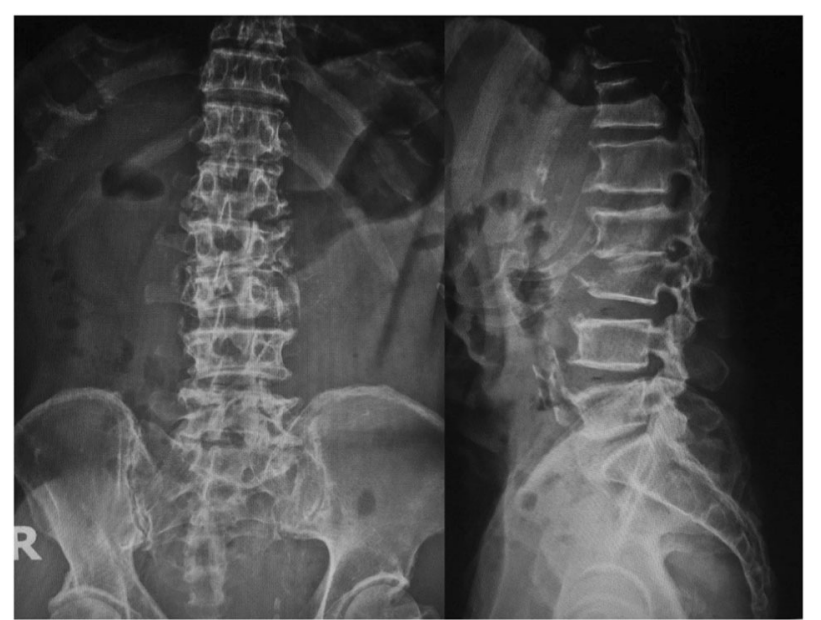

Fig. 1 Plain radiographs of lumbosacral spine in posteroanterior (left panel) and lateral (right panel) views showed osteolytic lesion with superior endplate destruction of L3 vertebra and vertebral collapse of L2 and L3 vertebral bodies. No abnormal paravertebral soft tissue shadow was demonstrated. Calcified abdominal aorta was seen 


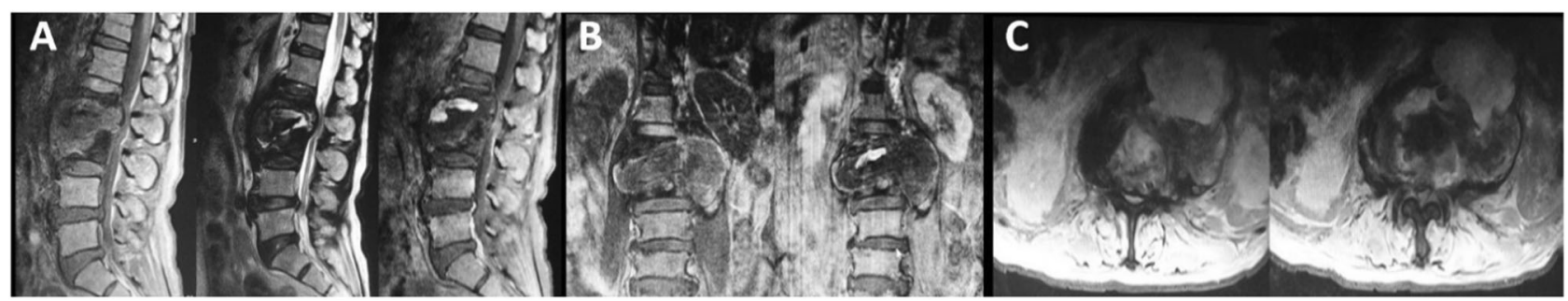

Fig. 2 Magnetic resonance imaging in mid-sagittal (a), mid-coronal (b) and axial (c) views revealed abnormal marrow signal intensity of L2-L3 vertebral bodies involving anterior to posterior column, anterior subligamentous spreading form L1 to L4, fluid collection at the

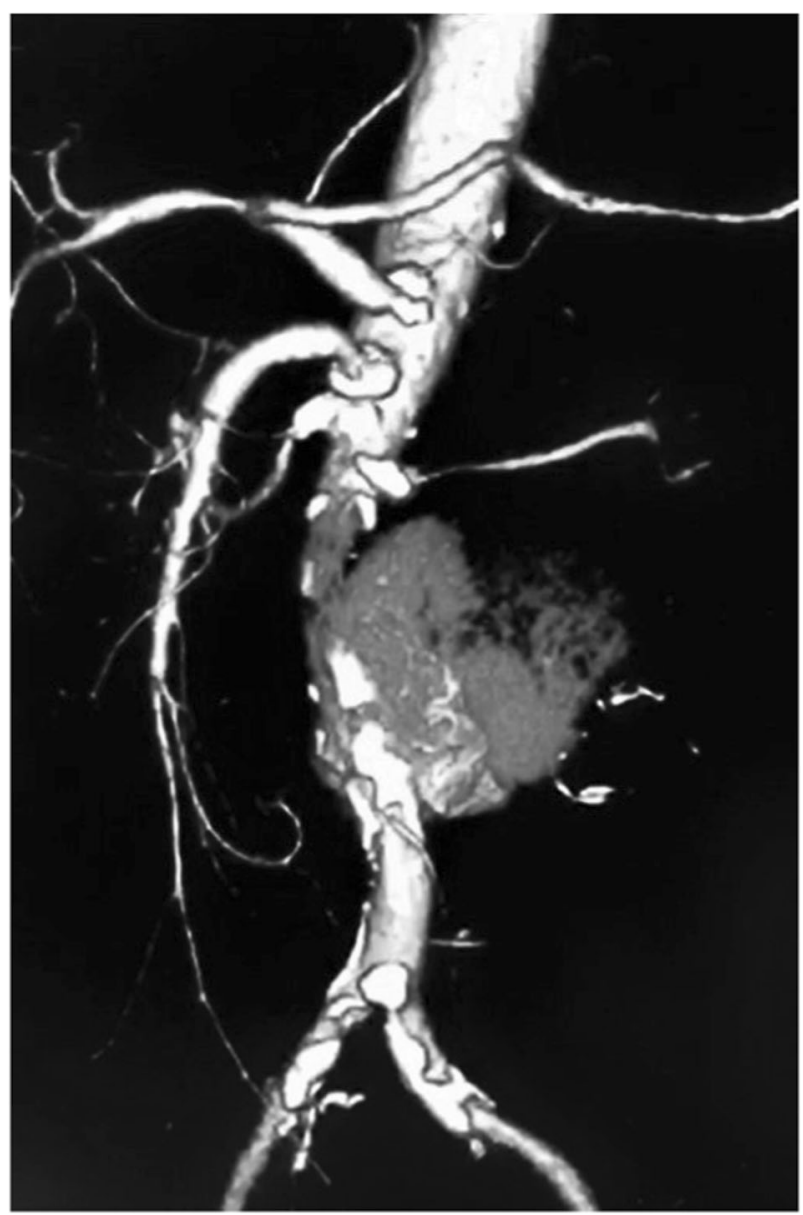

Fig. 3 Computerized tomographic angiography before performing endovascular aortic repair showed ruptured abdominal aneurysm at $30 \mathrm{~mm}$ below origin of the renal artery and $48 \mathrm{~mm}$ above aortic bifurcation

The patient underwent radical debridement via left transpsoas approach. During the operation, abdominal aortic aneurysm was iatrogenically ruptured (Fig. 3). Temporary packing was applied due to uncontrolled bleeding. After hemostasis was achieved, the ruptured aneurysm was emergently treated with an endovascular stent graft implantation using Endurant II AAA Stent Graft System via intervertebral disc L2/3 with the presence of paravertebral soft tissue mass formation extended and infiltrated to left psoas muscle at the same level

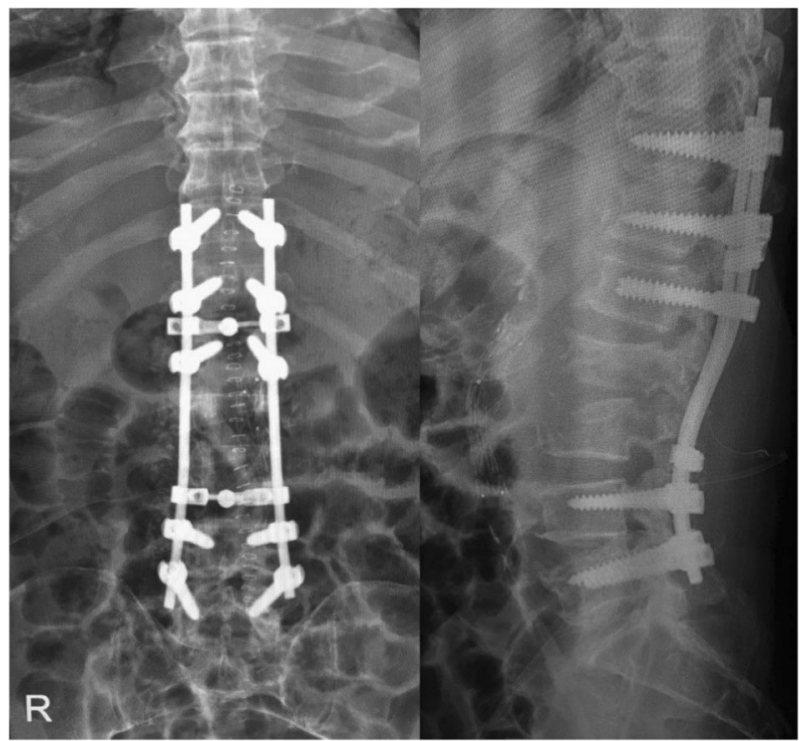

Fig. 4 Postoperative plain radiographs of the thoracolumbar spine in anteroposterior (left panel) and lateral (right panel) views showed posterior decompressive laminectomy L2-L3, fusion and instrumentation with pedicle screws and rods system at T11-L5. Endovascular stent graft implantation at abdominal aorta was seen

right common femoral artery. The patient remained hemodynamically stable and subsequently underwent a posterior decompressive laminectomy L2-L3, fusion and instrumentation with a pedicle screws and rods system at T11-L5 (Fig. 4). The surgically resected tissue was sent to the pathology department for histopathological examination and showed multiple pieces of fibrocollagenous tissue and scattered neutrophils. Few fragments of bone necrosis and granuloma were found. No evidence of tumor cells were seen. After surgery, the bilateral weakness of both lower extremity gradually improved and overall motor power and bowel bladder function were improved (awareness of the need to empty the bladder with improvement of voluntary anal sphincter contraction)(AIS category C; motor incomplete) $[8,9]$ (Table 1). The patient was continually treated with anti-tuberculous chemotherapy for 12 months. 


\section{Discussion}

Tuberculous spondylitis is one of the most common types of spinal tuberculosis, accounting for $60 \%$, followed by arachnoiditis (20\%), meningitis (12\%), and intramedullary lesion $(8 \%)[10,11]$. The most common pathogen of tuberculous spondylitis is Mycobacterium tuberculosis, a slow-growing aerobic organism with a growth-doubling time of about $20 \mathrm{~h}$ in conditions favorable to the bacillus [12]. Symptoms are usually slowly progressive and nonspecific and include fatigue, weight loss, fever, and chronic back pain. Neurologic symptoms are found on presentation in $75 \%$ of cases and include reduced sexual function, paraparesis, bowel-bladder dysfunction, and impaired autonomic regulation of organ functions [13]. The typical radiographic findings are destruction of the intervertebral disc space and the adjacent vertebral bodies, collapse of the spinal elements, and anterior wedging leading to the angular deformity and gibbus formation (palpable deformity because of involvement of multiple vertebra) [14]. Direct smear examination of tuberculous material obtained during surgery or by aspiration of the infected area may yield a positive result for tubercle bacilli if the sample contains more than $10^{5}$ bacilli $/ \mathrm{ml}$. However, the load of mycobacterium in the skeletal disease usually $<10^{5}$. Meanwhile, histopathologic studies can confirm the diagnosis only in $60 \%$ of cases. The most common cytological findings are epithelioid cell granulomas (90\%), granular necrotic background (83\%), and lymphocytic infiltration (76\%). Therefore, the best material for microbiological assessment appears to be the centrifuged residue material form a large quantity of abscess curetting from the walls of cold abscess and from the lining of the sinus tracts as close to the base as possible [12-14]. Due to the slow progression of clinical signs and symptoms, diagnosis is frequently delayed. In addition, the progression of the disease can lead to paravertebral and surrounding soft tissue invasion including the aorta and psoas muscle. The mechanisms of aortic involvement include hematogenous spread to the vasa vasorum, hematogenous seeding of atherosclerotic aortic plaques, and contiguous extension from periaortic structures such as the periaortic lymphatics, empyema cavities, and the spine [15, 16]. Surgical treatments for tuberculous spondylitis are indicated in the cases of signs and symptoms of cord compression, progressive impairment of pulmonary function, progression of kyphotic deformity, resistance to chemotherapy, and recurrence of the disease [1].

Mycotic or infected aortic aneurysm is also a rarity. These aneurysms can be divided into true aneurysm and false aneurysm or pseudoaneurysm. True aneurysm results from an inflammatory process longitudinally within the aortic wall promoting the formation of the aneurysm. However, the buildup of caseous necrosis of the aortic wall can result in luminal perforation, resulting in the formation of a pseudoaneurysm $[17,18]$. The prognosis is very poor as this condition has a tendency to grow rapidly and to rupture. The gold standard for treatment of this disease is resection of the aneurysm, extensive local debridement, and revascularization by in situ reconstruction or extra-anatomic bypass but carries high morbidity and mortality. In contrast, endovascular aortic repair (EVAR), which is a minimally invasive procedure and has less morbidity and mortality, is an alternative treatment [7, 19].

Mycotic or infected abdominal aortic aneurysm secondary to tuberculous spondylitis is extremely rare and lifethreatening. Few cases have been reported [16, 20-22]. Moreover, to our knowledge, the combined conditions of mycotic abdominal aortic aneurysm, tuberculous spondylitis, and cauda equina syndrome have not been previously reported in the literature. Precise diagnosis and expeditious treatment are the key to successful management. Treatment are to eradicate infection, prevent further neurological compromise, stabilize the spine and protect against aortic aneurysmal rupture. Surgical strategies are very important. In this case, the diagnosis of cauda equina syndrome was also made. Our initial strategy was first-stage radical surgical debridement, decompressive laminectomy, and stabilization of the spine with strut graft anteriorly and instrumentation with pedicle screws and rods system posteriorly, followed by the treatment of aortic aneurysm in the second stage. Unfortunately, an iatrogenic aortic aneurysm rupture occurred during performing radical debridement. So the surgical plan was changed to emergent implantation of endovascular stent graft. After the hemodynamics had been controlled, the patient subsequently underwent posterior decompressive laminectomy L2-L3, fusion and instrumentation with a pedicle screws and rods system at T11-L5. After surgery, the overall clinical outcomes were gradually improved and the patient was continually treated with antituberculous chemotherapy for 12 months.

In conclusion, concomitant mycotic aortic aneurysm and lumbar tuberculous spondylitis and psoas abscess with cauda equina syndrome is an extremely rare condition that requires prompt diagnosis and management. Its consequences can lead to serious complications such as permanent neurological damage, paralysis or death, if left untreated. The keys to successful management of this condition are accurate diagnosis and prompt treatment.

\section{Compliance with ethical standards}

Conflict of interest The authors declare that they have no conflict of interest. 


\section{References}

1. Camillo FX. Infections and Tumors of the Spine. Campbell's Operative Orthopaedics 13th edition. pp. 1824-52

2. Sai Kiran NA, Vaishya S, Kale SS, Sharma BS, Mahapatra AK. Surgical results in patients with tuberculosis of the spine and severe lower-extremity motor deficits: a retrospective study of 48 patients. J Neurosurg Spine. 2007;6:320-6.

3. Rasouli MR, Mirkoohi M, Vaccaro AR, Yarandi KK, RahimiMovaghar V. Spinal tuberculosis: diagnosis and management. Asian Spine J. 2012;6:294-308.

4. Kaufman SL, White RI Jr, Harrington DP, Barth KH, Siegelman SS. Protean manifestations of mycotic aneurysms. AJR Am J Roentgenol. 1978;131:1019-25.

5. Lee WK, Mossop PJ, Little AF, Fitt GJ, Vrazas JI, Hoang JK, et al. Infected (mycotic) aneurysms: spectrum of imaging appearance and management. Radiographics. 2008;28:1854-68.

6. Oderich GS, Panneton JM, Bower TC, Cherry KJ Jr, Rowland CM, Noel AA, et al. Infected aortic aneurysms: aggressive presentation, complicated early outcome, but durable results. J Vasc Surg. 2001;15:197-205.

7. Hsu RB, Chen RJ, Wang SS, Chu SH. Infected aortic aneurysms: Clinical outcome and risk factor analysis. J Vasc Surg. 2004;40:30-5.

8. American Spinal Injury Association. Reference Manual for the International Standards for Neurological Classification of Spinal Cord Injury. Chicago, IL: American Spinal Injury Association; 2003.

9. Krassioukov A, Biering-SØrensen F, Donovan W, Kennelly M, Kirshblum S, Krogh K, et al. International standards to document remaining autonomic function after spinal cord injury. J Spinal Cord Med. 2012;35:202-11.

10. Muthukunar N, Venkatesh G, Senthilbabu S, Rajbaskar R. Surgery for intramedullary tuberculoma of the spinal cord: report of 2 cases. Surg Neurol. 2006;66:69-74. discussion 74
11. Varghese P, Abdul Jalal MJ, Kandathil JC, Mathew IL. Spinal intramedullary tuberculosis. Surg J(NY). 2017;3:e53-7.

12. Agrawal V, Patgaonkar PR, Nagariya SP. Tuberculosis of the spine. J Craniovertebr Junction. 2010;1:74-85.

13. Falkensammer J, Behensky H, Gruber H, Prodinger WM, Fraedrich G. Successful treatment of a tuberculous vertebral osteomyelitis eroding the thoracoabdominal aorta: A case report. J Vasc Surg. 2005;42:1010-3.

14. Garg RK, Somvanshi DS. Spinal tuberculosis: A review. J Spinal Cord Med. 2011;34:440-54.

15. Hagino RT, Clagett GP, Valentine RJ. A case of Pott's disease of the spine eroding into the suprarenal aorta. J Vasc Surg. 1996;24:482-6.

16. Li FP, Wang XF, Xiao YB. Endovascular stent graft placement in the treatment of a ruptured tuberculous pseudoaneurysm of the descending thoracic aorta secondary to Pott's disease of the spine. J Card Surg. 2012;27:75-7.

17. Volini FI, Olfield RC Jr, Thompson JR, Kent G. Tuberculosis of the aorta. JAMA. 1962;181:78-83.

18. Golzarian J, Cheng J, Giron F, Bilfinger TV. Tuberculous pseudoaneurysm of the decending thoracic aorta: successful treatment by surgical excision and primary repair. Tex Heart Inst $\mathrm{J}$. 1999;26:232-5. Review

19. SÖrelius K, Mani K, BjÖrck M, Sedivy P, Wahlgren CM, Taylor $\mathrm{P}$, et al. Endovascular treatment of mycotic aortic aneurysms: a European multicenter study. Circulation. 2014;130:2136-42.

20. Dahl T, Lange C, Ødegard A, Bergh K, Osen S, Myhre HO. Ruptured abdominal aortic aneurysm secondary to tuberculous spondylitis. Int Angiol. 2005;24:98-101.

21. Takahashi Y, Sasaki Y, Shibata T, Suehiro S. Descending thoracic aorta aneurysm complicated with severe vertebral erosion. Eur J Cardiothorac Surg. 2007;31:941-3.

22. Hussein H, Azizi ZA. Tuberculous aortic pseudoaneurysm treated with in situ silver-impregnated vascular inlay graft. Asian J Surg. 2008;31:87-9. 